\title{
SEPARATION OF DIGITAL COMMUNICATION SIGNALS THROUGH JOINT SPACE-TIME DECORRELATION
}

\author{
J.Sala-Alvarez, G.Vázquez-Grau \\ Department of Signal Theory and Communications \\ Universitat Politècnica de Catalunya \\ Apt 30002, 08071 Barcelona,Spain \\ E-mail: alvarez@tsc.upc.es / gregori@tsc.upc.es
}

\begin{abstract}
${ }^{\dagger}$
In this paper we derive the optimum linear algorithm for the separation by an array of a given number of cyclostationary signals of the type encountered in digital communications. Spatially and temporally uncorrelated noise is considered at the input to each sensor. It is proved that the optimum signal separator can be expressed as a matrix transfer function in the z-domain. It is intended that this algorithm be used as a benchmark to test the performance of adaptive schemes. Therefore, bounds on the noise estimation covariance are obtained assuming perfect knowledge of the number of sources as well as of their timing and DOA
\end{abstract}

\section{INTRODUCTION}

The issue of separating digital communication signals (pseudo-random sequences that linearly modulate a shaping pulse) impinging on an array is examined under the scope of this paper. It is the purpose of this work to establish the fundamental performance bounds of any linear algorithm applied to the separation of the given signals. For the derivation of the optimum linear algorithm, we assume complete knowledge of all relevant parameters. It is understood than in practice, not all relevant information will be available and performance will be worse. In this way, we will be able to analyze any adaptive algorithm by comparing its performance with the bounds obtained in this paper. The main application intended for the results herein developed is performance analysis of source separation in multiple access communication systems. For the particular case of CDMA (Code Division Multiple Access) systems, each user modulates data on a pseudorandom sequence. When these signals are collected by an array and filtered, interference due to other users in the channel appears at the output of the filter matched to each user sequence (note that in the case of asynchronous

† This work has been supported by the National Research Plan of Spain CICYT, Grant Number TIC92-0800-C05-05. access, complete orthogonality between all the user spreading codes cannot be guaranteed for all possible interuser delays and hence multiaccess interference will always be present. Beamforming only will not succeed in separating each user as we may have that the number of users exceeds that of sensors). The assumption that the interference from other users adds up to white noise by the Central Limit Theorem is not valid as the user energy distribution may be far from uniform ${ }^{\dagger \dagger}$. Under these conditions, it is not unlikely that a very strong user can mask other weaker users. Therefore, the typical AWGN matched filter will not be optimum [1], [2].

Let us define the signal transmitted by each user in the following way:

$$
\begin{aligned}
s_{k}(n) & =\sum_{m=-\infty}^{m=+\infty} a_{k}(m) p_{k}^{\prime}\left(n-m N-\varepsilon_{k}\right) \\
1 \leq k \leq M & \\
& =\sum_{m=-\infty}^{m=+\infty} a_{k}(m) p_{k}(n-m N)
\end{aligned}
$$

where $\mathbb{A}_{k}=\left\{a_{k}(m),-\infty<m<+\infty\right\}$ constitutes the pseudorandom sequence transmitted by each source in a total of $M$ sources indexed by $k$. We have denoted the inter-user delays as the set $\left\{\varepsilon_{k}, 1 \leq k \leq M\right\}$. The set of pulselets $\mathbb{P}=$ $\left\{p_{k}(n-m N),-\infty \leq m \leq+\infty, 1 \leq k \leq M\right\}$ constitutes the support of the information transmitted by each user. Note that synchronization information is implicit in this set. Knowledge of $\mathbb{P}$ will be assumed throughout. These signals will be collected by an array, not necessarily linear. For the problem of interest we will assume that the narrow-band assumption is valid. Therefore, the signals received by each of the $L$ sensors can be expressed as,

$$
\underset{1 \text { sisL }}{r_{i}(n)}=w_{i}(n)+\sum_{k=1}^{k=M} S_{k i} s_{k}(n)
$$

$\dagger \dagger$ This is related to the Lindberg condition for the Central Limit Theorem. The limit distribution of the arithmetic mean of $\mathrm{N}$ random variables will approach a gaussian if the summation over the variances of the respective random variables diverges. This will not be the case when user energies are very dissimilar.

\section{IV-145}

0-7803-1775-0/94\$3.00 이 1994 IEEE 
The set $\mathbb{R}=\left\{r_{1}(n), 1 \leq i \leq L\right\}$ constitutes the available $L$ data sequences of the array from which the user symbols in $A_{k}$ have to be estimated and $W_{N}=\left\{w_{i}(n), 1 \leq i \leq L\right\}$ are the interfering AWGN sequences. $S_{k i}$ denote the components of the steering vector associated to each user. The components of the steering matrix $\mathbf{S}$ can be expressed in terms of the steering vectors of each user as,

$$
\mathbf{S}=\left[\mathbf{s}_{1}, \mathbf{s}_{2}, \ldots, \mathbf{s}_{M}\right], \mathbf{s}_{k}^{1}=\left[e^{j \theta_{k, 1}}, e^{j \eta_{k, 2}}, \ldots, e^{j 0_{k, 2}}\right]
$$

with $\theta_{\mathrm{kl}}$ the wavefront phase shifts of every sequence impinging on the array. We can now group the sensor signals $r_{i}(n)$ and $w_{i}(n)$ in Eq.2 into the vectors $r(n)$ and $w(n)$, and the symbol seyuences $a_{k}(n)$ and the pulses $p_{k}(n)$ in Eq. $l$ into the vectors $\mathbf{a}(n)$ and $p(n)$. This permits us to express $r(n)$ in terms of a $(n)$ in very compact notation,

$$
\mathbf{r}(n)=\mathbf{w}(n)+\mathbf{S} \sum_{m=-\infty \infty}^{m=+\infty} \mathbf{p}(n-m N) \bullet \mathbf{a}(m)
$$

where - denotes the Schur product (component-wisc) between rectangular matrices. The noise process $w(n)$ is considered temporally and spatially uncorrelated, with $\mathrm{E}\left[\mathbf{w}(\mathrm{n}) \mathbf{w}^{\mathrm{H}}(\mathrm{m})\right]=\mathbf{I} \delta_{\mathrm{nm}}$.

The optimum linear algorithm for the separation of $M$ signals of the type defined in $\mathrm{Eq} l$ will be derived on a least square error criterion and its performanec evaluated. A fundamental condition to make separation possible is that the sequences in JP be linearly independent. It is guaranteed in this way that the information of each user can be recovered by means of a linear algorithm. Just view each sequence in $\mathbb{P}$ as a vector whose component is precisely it user's symbol at a given time.

Advantage is taken both from decorrelation in the spatial (index $\mathrm{i}$ in Eq 2) and in the time (index $\mathrm{n}$ in Eq. 1) domain. In this sense, a number of signals which is larger than the dimension of the observation vector (equal to the number of sensors if we are considering an array), $\mathrm{M} \geq \mathrm{L}$, can be extracted from the available dati. That is, although for this case separation cannot be achieved with spatial processing only, the fact that each uscr has been assigned a different waveform permits some temporal post-processing that guarantees reliable separation.

The structure of the stecring malrix bears heavily on the capability of the system to separate all signals. It can be shown that better decorrelation may be achicved among the involved signals depending on how orthogonal the columns of the steering matrix are. The orthogonality or the linear independence among the columns of the steering matrix $S$ is quantified by the relative magnitude (with respect to the diagonal elements) of the off-diagonal components of the associated Grammian matrix $\mathbf{S}^{\mathrm{H}} \mathbf{S}$.

In general, a non-zero correlation among signals, temporally and spatially, leads to a decreased performance in the scparation capability of the optimum algorithm.
This can be explained in terms of the equivalence between cross-corrclation and partial superposition of the subspaces associated to each particular signal. In the limiting case when the cross-correlation between two of the signals attains its maximum value, the associated subspaces have become parallel and the separation problem is rankdefficient. That is, there is no way of linearly recovering each of those two signals unless some side information is available.

\section{ALGORITHM DERIVATION}

The optimization criterion is defined as the minimization of the statistical expectation of the following error norm,

$$
L=\mathrm{E}\left\{\mathbf{e}_{r}^{\prime \prime}(n) \mathbf{e}_{r}(n)\right\}, e_{r}(n)=\mathbf{r}(n)-\mathbf{r}_{a}(n)
$$

with $\mathbf{r}$ denoting the vector of signals receved at each sensor at a given time and $r_{\mathrm{a}}$ the estimate of the noiseless signal. For the minimization of $L$, we will take a finite record and then let its lenglh $N^{\prime}$ go to infinity,

$$
L_{N^{\prime}}=\frac{1}{2 N^{\prime}+1} \sum_{n=-N^{\prime}}^{n=+N^{\prime}} \mathrm{e}_{r}^{\prime \prime}(n) \mathrm{e},(n)
$$

For minimization of this expression we take derivatives with respect to $\mathbf{a}^{\mathrm{H}_{(}}\left(m^{\prime}\right)$ and equatc to zero,

$$
\left(2 N^{\prime}+1\right) \frac{\partial}{\partial \mathbf{a}^{\prime \prime}\left(m^{\prime}\right)} L_{N^{\prime}}=-\sum_{n=N^{\prime}}^{n+N^{\prime}} \frac{\partial \mathbf{r}_{a}^{\prime \prime}(n)}{\partial \mathbf{a}^{\prime \prime}\left(m^{\prime}\right)} \mathbf{e}_{r}(n)=0
$$

Using some propertics of the Schur product ${ }^{\dagger}$ and Eq 4 to obtain a model for $\boldsymbol{r}_{\mathrm{a}}$, the derivative term and $\mathbf{r}_{\mathrm{a}}$ can be expanded into Eq. 7 to yicld.

$$
\begin{aligned}
& \frac{\partial \mathbf{r}_{\mathrm{a}}^{\mathrm{H}}(n)}{\partial \mathbf{a}^{\mathrm{H}}\left(m^{\prime}\right)}=\operatorname{diag}\left(\mathbf{p}^{\prime \prime}\left(n-m^{\prime} N\right)\right) \mathbf{S}^{\prime \prime} \Rightarrow \\
& \mathbf{b}\left(m^{\prime}\right)=\sum_{n} \operatorname{diag}\left(\mathbf{p}^{\mathrm{H}}\left(n-m^{\prime} N\right)\right) \mathbf{S}^{\prime \prime} \mathbf{r}(n) \\
& =\sum_{n} \operatorname{diag}\left(\mathbf{p}^{\mathrm{H}}\left(n-m^{\prime} N\right)\right) \mathbf{S}^{\mathrm{H}} \mathbf{S} \sum_{m} \mathbf{p}(n-m N) \bullet \mathbf{a}(m)
\end{aligned}
$$

The sequence $\mathbf{b}\left(m^{\prime}\right)$ in Eq. 7 constitutes a set of sufficient statistics for the estimation of the user symbols. If we recast $\mathbf{b}\left(\mathrm{m}^{\prime}\right)$ in the following form, we can see that $\mathbf{b}\left(\mathrm{m}^{\prime}\right)$ is oblained by passing through a matched filter the scquence resulting from the scalar multiplication of the vector of input sensor signals by the steering vector of cach user,

$$
\begin{aligned}
& b_{k}\left(m^{\prime}\right)=\sum_{n=\ldots N^{*}}^{n=+N^{*}}\left(s_{k}^{\prime \prime} r(n)\right) p_{k}\left(n-m^{\prime} N\right) \\
& 1 \leqslant k \subset M
\end{aligned}
$$

11) $\mathbf{a} \cdot \mathbf{b}=\operatorname{diag}(\mathbf{a}) \mathbf{b}=\mathbf{b} \cdot \mathbf{a}$

2) $\operatorname{diag}(\mathbf{a})$ Adiag $(\mathbf{b})=\left(\mathbf{a} \mathbf{b}^{\mathrm{T}}\right) \cdot \mathbf{A}$ 
Continuing with Eq.8, the right-hand side term can be simplified as follows,

$$
\begin{aligned}
& \sum_{n} \operatorname{diag}\left(\mathbf{p}^{\mathrm{H}}\left(n-m^{\prime} N\right)\right) \mathbf{S}^{\mathrm{H}} \mathbf{S} \sum_{m} \mathbf{p}(n-m N) \bullet \mathbf{a}(m) \\
& =\sum_{m} \mathbf{a}(m) \sum_{n} \operatorname{diag}\left(\mathbf{p}^{\mathrm{H}}\left(n-m^{\prime} N\right)\right) \mathbf{S}^{\mathrm{H}} \mathbf{S} \operatorname{diag}(\mathbf{p}(n-m N)) \\
& =\sum_{m} \mathbf{a}(m) \sum_{n}\left(\mathbf{p}^{*}\left(n-m^{\prime} N\right) \mathbf{p}^{\mathrm{T}}(n-m N)\right) \cdot\left(\mathbf{S}^{\mathrm{H}} \mathbf{S}\right)
\end{aligned}
$$

In its final form, we have that the sequence of sufficient statistics $\mathbf{b}\left(\mathrm{m}^{\prime}\right)$ is related to the sequence of user symbol estimates $\mathbf{a}\left(m^{\prime}\right)$ by way of the following cross-correlation matrices,

$$
\mathbf{b}\left(m^{\prime}\right)==\sum_{m=-\infty}^{m=+\infty}\left(\mathbf{R}\left(m, m^{\prime}\right) \cdot\left(\mathbf{S}^{\mathrm{H}} \mathbf{S}\right)\right) \mathbf{a}(m)
$$

The components of $\mathbf{R}\left(m, m^{\prime}\right)$ are just the finite crosscorrelation of the sequences $p_{k}\left(n-m^{\prime} N\right)$ and $p_{k}(n-m N)$,

$$
\left[\mathbf{R}\left(m, m^{\prime}\right)\right]_{k k^{\prime}}=\sum_{n=-N^{\prime}}^{n=+N^{*}} p_{k}^{*}\left(n-m^{\prime} N\right) p_{k^{\prime}}(n-m N)
$$

Note that as the summation limits in Eq. 12 are finite, it does not hold that $\mathbf{R}\left(m, m^{\prime}\right)$ are stationary correlation matrices with $\mathbf{R}\left(m, m^{\prime}\right)=\mathbf{R}\left(m-m^{\prime}\right)$.

The components of $\mathbf{R}\left(m, m^{\prime}\right)$ are weighted through the Schur product by the Grammian of the steering matrix, $\mathbf{S}^{\mathrm{H}} \mathbf{S}$. This proves to be a nice way of relating the global correlation among users with their temporal and spatial correlation, respectively. The global decorrelation can be quantified by the relative magnitude of the off-diagonal terms of the following matrices with respect to their diagonal terms,

$$
\overline{\mathbf{R}}\left(m, m^{\prime}\right)=\mathbf{R}\left(m, m^{\prime}\right) \cdot\left(\mathrm{S}^{\prime \prime} \mathrm{S}\right)
$$

As the global correlation matrices are built by Schur product of two other correlation matrices, global decorrelation will always be smaller than either temporal or spatial decorrelation. When any off-diagonal term of either $\mathbf{R}$ or $\mathbf{S}^{\mathrm{H}} \mathbf{S}$ is zero, that is, we have full temporal decorrelation (orthogonal modulation sequences) or spatial decorrelation (orthogonal steering vectors), full global decorrelation is assured.

Note that although $\mathbf{b}\left(m^{\prime}\right)$ in Eq. 8 is a set of sufficient statistics, interference from other users is still present in each component of $\mathbf{b}\left(\mathrm{m}^{\prime}\right)$. The beamforming stage will not be capable of decoupling all users for two reasons: first, the steering vectors are not necessarily orthogonal and second, there may be more sources than sensors. Therefore, some post-processing is necessary. On these grounds, we need to invert the system in Eq.11 to obtain the desired sequence $a\left(m^{\prime}\right)$. Let us take $z$ transforms in Eq. 11 after letting $\mathrm{N}^{\prime}$ go to infinity, we then have,

$$
\mathbf{B}\left(z^{-1}\right)=\left(\sum_{n=-\infty}^{n=+\infty} \overline{\mathbf{R}}(n) z^{-n}\right) \mathbf{A}\left(z^{-1}\right)
$$

Where we have used this property of the finite crosscorrelation matrices: $N^{\prime} \rightarrow \infty \Rightarrow \mathbf{R}\left(m, m^{\prime}\right)=\mathbf{R}\left(m-m^{\prime}\right)$. The inversion of this system is called the decorrelation stage and is depicted in Figure 1. Under these conditions, the decorrelator can be viewed as a time-invariant, noncausal vectorial filter whose matrix transfer function is given straightforwardly by,

$$
\Delta\left(z^{-1}\right)=\left[\sum_{n=-\infty}^{n=+\infty} \overline{\mathbf{R}}(n) z^{-n}\right]^{-1}
$$

Note that the cyclostationary character of the signal is modified after the decimation stage, making it stationary. No information is lost through decimation as the vectors fed into the decorrelation stage constitute a set of sufficient statistics.

It can be shown that stability of the vector filter is guaranteed whenever the matrix $\Delta\left(\mathrm{e}^{-j w}\right)$ is strictly positive definite on the unit circle, $\omega$ in $[-\pi,+\pi]$ : No (positive) eigenvalue of $\Delta\left(e^{-j w}\right)$ can become zero. An equivalent condition is that no root of the determinant dets $\Delta^{-1}\left(z^{-1}\right)$ may be found on the unit circle. Evaluation of the covariance matrix of this estimator reveals that the elements along the diagonal of $\Delta\left(e^{-j w}\right), \Delta_{k k}\left(e^{-j w}\right)$, are precisely the power spectra of the estimation noise of the $\mathrm{k}$-th PN sequence. The associated correlation function of the estimation noise vector, $\mathbf{n}_{\mathbf{a}}(\mathrm{n})$ is precisely the decorrelator's matrix transfer function, $\Delta\left(\mathrm{e}^{-\mathrm{jw}}\right)$. Therefore, the variance of the estimation error of each particular symbol fulfils.

$$
\sigma_{k}^{2}=\frac{1}{2 \pi} \int_{-\pi}^{+\pi} \Delta_{k k}\left(e^{-j \omega}\right) d \omega
$$

\section{SIMULATIONS}

In the simulations, a particular case of eight signals impinging on a two sensor array has been considered. In Figure $3 \mathrm{wc}$ have depicted the estimation noise power spectrum at the output of the decorrelator $\Delta$ corresponding to one of the signals for three cases: a one sensor array, a two-sensor array and a one-sensor array with no other signals present (conventional matched filter). Note that the presence of other signals not orthogonal to the sequence of interest results in coloured estimation noise. The lowest covariance is obtained when all scquences are mutually orthogonal, then, no decorrelation is necessary and the estimation noise spectrum becomes white.

In Figure 1 we have depicted a schematic diagram of the proposed system. At the input to the filter bank, the $\mathrm{L}$ input signals are combined according to the hermitian of the steering matrix $\mathbf{S}$, as was derived in Eq.9. 


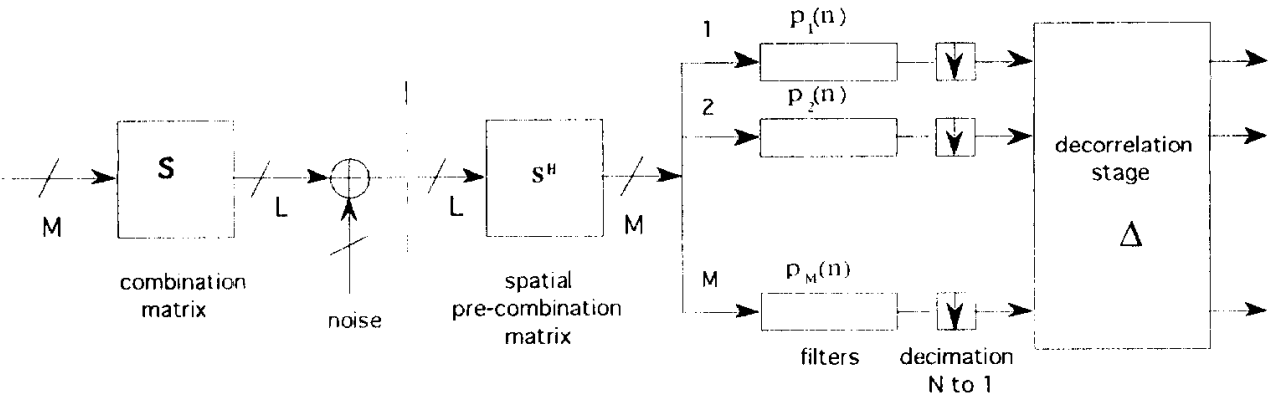

Figure 1

Derived algorittm for signal separation

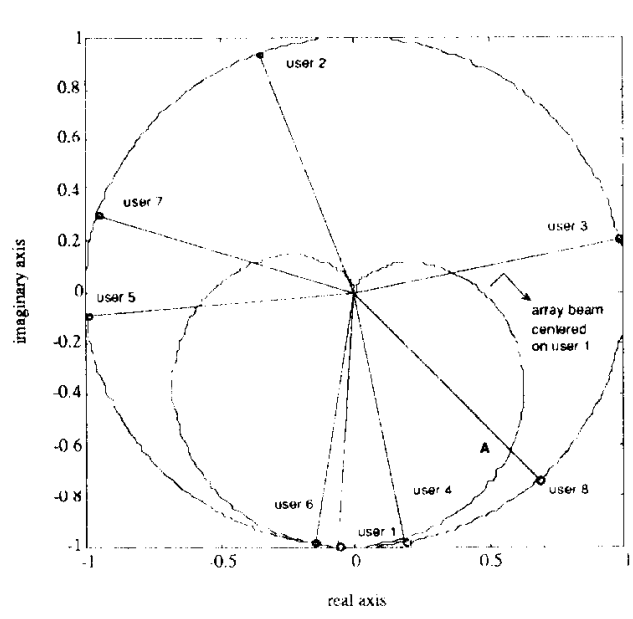

Figure 2

Depiction of the DOAs of 8 users for the given scenario. The DOAs are represented as a phasor whose angle is the phase shift between sensors experimented by the corresponding user's wavefront impinging on the array. Therefore, all DOAs appear as hollow dots on the unit circle. The angles 0 and $\pi$ correspond to the broadside and endfire look directions of the array, respectively. The array beam gain with respect to the inter-sensor phase shift has been superimposed, with the maximum gain normalized to one and focused on user 1 . The length of the intersection of the DOA lines with the array beam centered on the user of interest yields the scalar product of the respective sleering vectors, normalized to the number of sensors (the distance between point $A$ and the origin, for user 8), the components of the Grammian of the stecring matrix $\mathbf{S}$. It can be appreciated that when the array is focused on user 1 , only user 2 has been reliubly filtered out in the beamforming stage.

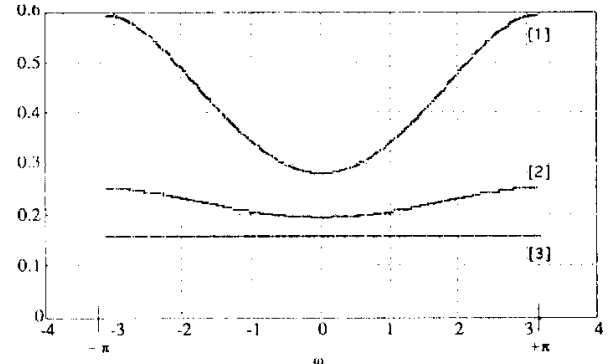

Figure 3:

(1)power spectrum of estimation noise for a one sensor anray: $\Delta_{k k}\left(e^{-j w}\right), L=1(2)$ lwice the power spectrum for a two-sensor array: $2 \Delta_{k k}\left(e^{-j w}\right), L=2$ (3)power spectrum for a one sensor array in the presence of a single signal.

\section{REFERENCES}

[1] Ruxandra Lupas, Sergio Verdú, "Near-Far Resistance of Multi-User Detectors in Asynchronous Channels ", IEEE Transactions on Communications., vol 38, pp 4965()8, April 1990.

[2] Sergio Verdú, "Multiuser Demodulation ". Proceedings of the Third International Workshop on Digital Signal Processing Techniques Applied to Space Communications. ESTEC, Noordwijk, the Netherlands. Session 3. pp.18-36. 23-25 September 1992.

[13] Simon Haykin, "Adaptive Filter Theory ", Appendix A, "Complex variables", pp 770-781. Prentice-Hall Inc., 1991.

[4] Louis L. Scharf, "Statistical Signal Processing ". Addison Wesley Pub. Co. 1991.

[5] Kohno, R.; Imai, H; Hatori, M.; Pasupathy, S., "Combinations of an Adaptive Array Antenna and a Canceler of Interference for Direct-Sequence SpreadSpectrum Multiple-Access Systems ", IEEE Journal on Selected Arcas in Communications, vol. 8, pp 675-82. May 1990 